\title{
Beneficial effects of percutaneous minimally invasive surgery for patients with fractures in the thoracic spine
}

\author{
XIN WANG ${ }^{1,2}$, YANG LIU $^{2}$, XINWEI WANG ${ }^{2}$, HUAJIANG CHEN $^{2}$, PENG CAO $^{2}$, \\ YE TIAN $^{2}$, XIAOYU WU ${ }^{2}$, YU CHEN ${ }^{2}$ and WEN YUAN ${ }^{2}$ \\ ${ }^{1}$ Department of Orthopedics, First Clinical Medical College of Lanzhou University, \\ The First Hospital of Lanzhou University, Lanzhou, Gansu 730000; ${ }^{2}$ Department of Orthopedics, \\ Changzheng Hospital Affiliated to The Second Military Medical University, Shanghai 200003, P.R. China
}

Received April 21, 2017; Accepted September 6, 2017

DOI: $10.3892 /$ etm.2018.6887

\begin{abstract}
Percutaneous minimally invasive surgery (PMIS) is the most common surgical procedure used in patients with fractures in the thoracic spine. In the present study, the benefits of PMIS compared with the conventional open surgery (COS) were investigated in patients with thoracic spine fractures. A total of 84 patients were recruited in the current analysis. Inflammatory responses were measured in all patients subsequent to PMIS and COS. It was demonstrated that PMIS produced reduced inflammatory responses as compared with COS in clinical patients. In addition, the results revealed that kyphosis, anterolisthesis and the neurological state were significantly improved in patients subjected to PMIS compared with those receiving $\operatorname{COS}(\mathrm{P}<0.01)$. The mean values of bone reduction and fracture correction loss were similar in the PMIS and COS groups (4.5 degrees). Furthermore, the outcomes indicated that the sensitivity for fracture localization to the correct vertebra was 0.90 in PMIS and 0.92 in COS $(\mathrm{P}>0.05)$. It was observed that patients who had undergone PMIS required reduced hospitalization time compared with the COS-treated patients $(\mathrm{P}=0.026)$. Additionally, the mean bleeding volume during surgery was $538 \mathrm{ml}$ in the PMIS patients and $845 \mathrm{ml}$ in the COS patients. Regarding physical activity, patients who underwent PMIS were relative more active compared with the COS patients $(\mathrm{P}=0.038)$ in a 14-day observation. In conclusion, the current clinical analysis indicated that PMIS was more beneficial for the treatment of patients with fractures in the thoracic spine, and the study provided further evidence for the management thoracic spine injuries.
\end{abstract}

Correspondence to: Dr Wen Yuan, Department of Orthopedics, Changzheng Hospital Affiliated to The Second Military Medical University, 415 Fengyang Road, Shanghai 200003, P.R. China

E-mail: yuanwenspine1@163.com

Key words: percutaneous minimally invasive surgery, fractures in the thoracic spine, conventional open surgery, inflammation, physical activity

\section{Introduction}

Fractures in the thoracic spine are a result of thoracolumbar bone damage caused by external forces (1). These injuries frequently occur in young patients with high-energy injuries caused by car accidents, falling from a height and other accidents (2). Merging neural function damage is one of the most common complications in patients with thoracolumbar fractures, which frequently results in great difficulties and challenges in treating these patients (3). Singh et al (4) have demonstrated that cervical and lumbar spine injuries and rib fractures are significantly associated with the causes of thoracic spine injury. Additionally, traumatic thoracic spine fractures often lead to inter- and intraobserver injury of vertebral, local and segmental kyphosis in lateral X-rays, and appropriate studies should be performed prior to providing general recommendations by surgeons $(5,6)$.

Percutaneous minimally invasive surgery (PMIS) is a small trauma surgery performed using an auxiliary laparoscope, thoracoscope and other modern medical instruments to perform the surgery $(7,8)$. PMIS is the most common surgical procedure used for patients with fractures in the thoracic spine (9). A meta-analysis has indicated minimally invasive direct coronary artery bypass graft surgery or percutaneous coronary intervention for proximal left anterior descending artery stenosis may decrease the risk for target vessel interventions (10). Kwan et al (11) have also demonstrated that minimally invasive spinal stabilization combined with the use of fluoroscopic-guided percutaneous screws can be regarded as a type of palliative surgery in patients with spinal metastasis. In addition, PMIS presents more advantages compared with open spine surgery in the treatment of fractures of the thoracolumbar junction, as suggested by a comparative effectiveness review (12). Furthermore, PMIS combined with all methods, including pedicle screw fixation and vertebroplastry with grafting has been investigated in preventing grafting for new thoracolumbar burst fractures in a previous study (13).

In the present study, the benefits of PMIS and conventional open surgery (COS) in the treatment of patients with fractures in the thoracic spine were compared. The study observed that PMIS decreased the postoperative infection rate and inflammatory responses. It was also demonstrated that the kyphosis, 
anterolisthesis, neurological state and hospitalization time were significantly improved in patients with fractures in the thoracic spine subsequent to PMIS.

\section{Materials and methods}

Patients. The current clinical trial (approval number, CZHA2010M0430) was conducted in strict accordance with the recommendations provided in the Guide for the Care and Use of clinical study of Pharmaceutical Administration Measures for Implementation (14). The current study was approved by the ethics committee of Changzheng Hospital Affiliated to the Second Military Medical University. All patients were required to provide written informed consent prior to participation in the current study. In total, 84 patients with fractures in the thoracic spine (T4-10) who were surgically treated at Changzheng Hospital Affiliated to the Second Military Medical University (Shanghai, China) between May 2010 and June 2015 were enrolled into the present study. Exclusion criteria for patients with fractures in the thoracic spine were based on the diagnostic outcomes of radiographs, computed tomography scan and magnetic resonance imaging (15). The sensitivity of PMIS and COS for fracture localization was determined by the actual fracture to fracture diagnosed by PMIS or COS as described previously (16).

ELISA. Blood samples $(10 \mathrm{ml})$ were obtained from patients with fractures in the thoracic spine on day 7 after PMIS or COS. Serum was obtained from blood samples after centrifugation at $4,000 \mathrm{x} \mathrm{g}$ for $10 \mathrm{~min}$ at $4^{\circ} \mathrm{C}$ The serum levels of interleukin (IL)-1 (cat no. 88-7261-22; Thermo Fisher Scientific, Inc., Waltham, MA, USA), IL-17 ((cat no. BMS2037-2; Thermo Fisher Scientific, Inc.), tumor necrosis factor $\alpha$ (TNF- $\alpha$; KHC3014; Thermo Fisher Scientific, Inc.) and IL-6 (KHC0062; Thermo Fisher Scientific, Inc.) were analyzed using ELISA kits, according to the manufacturer's protocol. Subsequently, the serum concentration levels of IL-1, IL-17, TNF- $\alpha$ and IL-6 were measured by an enzyme microplate reader at $450 \mathrm{~nm}$.

Analysis of physical activity. The physical activity of patients with fractures in the thoracic spine on day 7 after PMIS or COS was measured as described previously (17). Briefly, following the PMIS or COS, the patients received a functional training program initiated by the nurses in addition to the usual physiotherapy. The functional training program included walking in the corridor for $30 \mathrm{~min}$ each day, and was targeted to the level of difficulty relevant for each participant.

Analysis of visual analog scale (VAS) and Japanese Orthopedic Association (JOA) scores. The VAS and JOA scores for patients with fractures in the thoracic spine were investigated 7 days following PMIS or COS. The patients were requested to report their pain levels using a $10-\mathrm{cm}$ VAS scoring system (18). All patients were able to self-rate their pain. Furthermore, the JOA score was used to evaluate the clinical neurological symptoms and neurological status of the patients as described in a previous study (19).

Anterolisthesis and neurological state. Anterolisthesis was observed in patients with fractures in the thoracic spine on day 7 after PMIS or COS (20). Briefly, the neurological state of patients who had undergone PMIS or COS was determined using the American Spinal Injury Association (ASIA) scale, as described previously (21). Each patient's neurologic status was classified between grade 1 (complete paraplegia) and grade 5 (normal neurologic status). The presence of kyphosis and anterolisthesis was assessed according to radiographic deformities (22). The reduction and correction loss of patients with fractures after PMIS or COS was analyzed as described previously (23).

Statistical analysis. The data are expressed as the mean \pm standard error in each experiment. Statistical differences between groups were assessed using one-way analysis of variance in six replicate experiments, along with the post-hoc Dunnett's test. $\mathrm{P}<0.05$ was considered to indicate a statistically significant difference.

\section{Results}

Characteristics and inflammation of patients with fractures in the thoracic spine subsequent to receiving PMIS and COS. CT scanning confirmed the location of the fractures in the thoracic spine of patients before and after surgery of PMIS or COS (Fig. 1). In total, 48 patients $(84 \%)$ were men and 36 $(16 \%)$ were women, with a mean age of $32.60 \pm 10.28$ years. A total of 42 patients received PMIS and 42 patients received COS. The characteristics of patients are listed in Table I. On day 7 after PMIS or COS, the serum levels of inflammatory cytokines in the patients were analyzed by ELISA. As shown in Fig. 2A-D, the IL-1, TNF- $\alpha$, IL-17 and IL-6 serum levels were significantly lower in PMIS patients when compared with the COS patients $(\mathrm{P}<0.01)$. These results indicate that the inflammatory risk was reduced following PMIS compared with COS for patients with fractures in the thoracic spine.

Comparison of hospitalization time for fracture localization between patients with fractures in the thoracic spine receiving PMIS and COS. The results demonstrated that the sensitivity for fracture localization to the correct vertebra was 0.90 in PMIS and 0.92 in COS, and was not significantly different between the two groups (Fig. 3A; P>0.05). In addition, the mean values of reduction and correction loss were similar in the PMIS and COS groups (Fig. 3B; 4.5 degrees; $\mathrm{P}>0.05$ ). It was also observed that patients who underwent PMIS required a markedly reduced hospitalization time compared with patients who underwent COS (Fig. 3C; $\mathrm{P}=0.026$ ). These observations indicate that PMIS is beneficial in reducing the hospitalization time following surgery in patients with thoracic spine fractures.

Comparison of kyphosis, anterolisthesis, neurological state and physical activity following PMIS or COS in patients with fractures in the thoracic spine. As shown in Fig. 4A, the kyphosis score was significantly improved in patients subsequent to PMIS as compared with patients who received $\operatorname{COS}$ (Fig. 4A; $\mathrm{P}<0.05$ ). It was also observed that the anterolisthesis and neurological state were significantly improved in patients receiving PMIS as compared with patients receiving COS (Fig. 4B and $\mathrm{C} ; \mathrm{P}<0.01$ ). Notably, regarding the level 

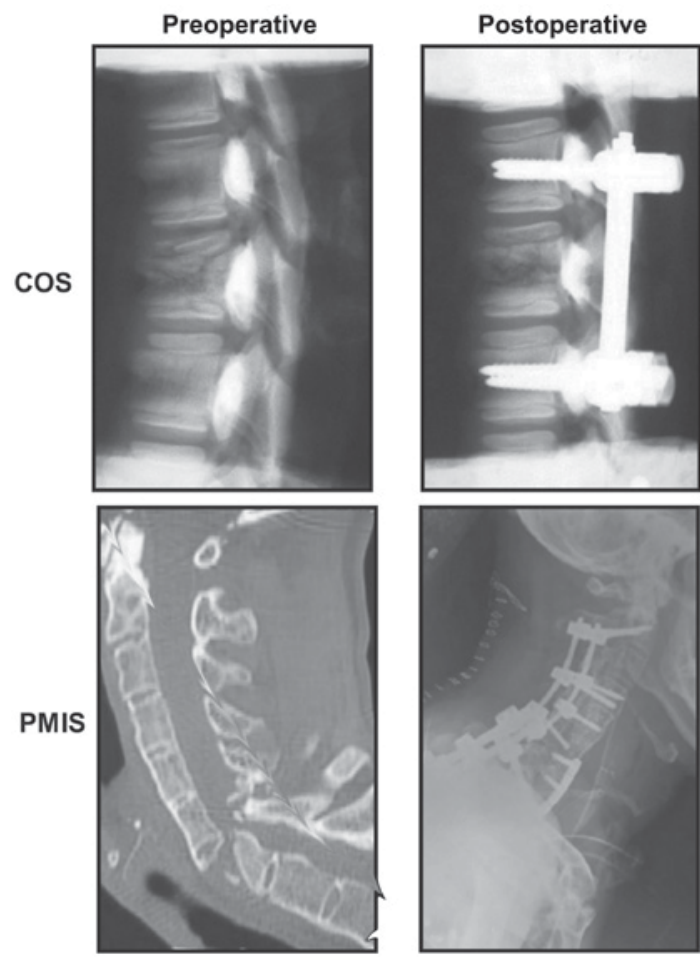

Figure 1. Representative images of patients with fractures in the thoracic spine prior to and post treatment with PMIS and COS determined by computed tomography. PMIS, percutaneous minimally invasive surgery; COS, conventional open surgery.

of physical activity, patients who had undergone PMIS were evidently more active when compared with the COS patients $(\mathrm{P}<0.05$; Fig. 4D). These findings indicate that PMIS was able to increase the physical activity of patients with fractures in the thoracic spine as compared with the COS procedure.

Comparison of physical condition following PMIS or COS in patients with fractures in the thoracic spine. In the present study, the mean follow-up period of patients was 6 months. The mean surgery duration was $\sim 170$ min (range, 120-294 min) in the PMIS and COS groups, which was not significantly different the two groups $(\mathrm{P}=0.870$; Fig. 5A). It was observed that the mean bleeding volume during surgery was $538 \mathrm{ml}$ in the PMIS group and $845 \mathrm{ml}$ in the COS group, with a significantly reduced blood loss observed during PMIS (Fig. 5B; $\mathrm{P}=0.023$ ). Furthermore, the mean JOA and VAS scores in patients who had undergone PMIS were significantly improved in comparison with those in patients who had undergone COS (Fig. 5C and D; $\mathrm{P}<0.01$ ). Collectively, these results suggest that PMIS presented a superior performance when compared with $\mathrm{COS}$ in patients with fractures in the thoracic spine.

\section{Discussion}

Traumatic fractures in the thoracic spine are common worldwide, and are derived from a strong impact force damaging the sternum (24,25). PMIS is a type of minimally invasive surgery performed using a laparoscope, thoracoscope or endoscope, which causes fewer injuries and surgery-associated defects as compared with the COS procedure $(8,26)$. It has been suggested that PMIS presents certain advantages for the treatment of
Table I. Characteristics of patients with fractures in the thoracic spine $(n=84)$.

\begin{tabular}{lcc}
\hline Characteristics & Men & Women \\
\hline Number & 48 & 36 \\
Age range, years & $22.3-54.8$ & $24.6-58.2$ \\
Cause of injury, $\mathrm{n}$ & & \\
Car accident & 32 & 21 \\
Fall from height & 16 & 15 \\
Site of fracture, $\mathrm{n}$ & - & - \\
T4/T5 & 10 & 8 \\
T6/T7 & 12 & 12 \\
T8/T9 & 14 & 7 \\
T9/T10 & 12 & 9 \\
COS & 24 & 18 \\
PMIS & 24 & 18 \\
\hline
\end{tabular}

patients with fractures in the thoracic spine $(13,27)$. Thus, the present study compared the differences between PMIS and COS performed in patients with fractures in the thoracic spine. The results indicated that the inflammatory risk and hospitalization time were significantly reduced subsequent to PMIS as compared with COS in patients with fractures in the thoracic spine. It was also observed that the physical activity, bleeding volume, as well as the mean JOA and VAS scores, were markedly improved in patients undergoing PMIS compared with those in patients undergoing COS.

A previous study has indicated the PMIS is a reliable method in fracture management (28). Pan et al (29) have also indicated that treatment of children with supracondylar fracture of the humerus using PMIS decreased the recovery time, hospitalization days and blood transfusion rate. In addition, minimally invasive posterior decompression combined with percutaneous pedicle screw fixation has been demonstrated to achieve a similar effect with COS for the treatment of thoracolumbar fractures with neurological deficits (27). Furthermore, the efficacy and safety of simultaneous combined minimally invasive percutaneous nephrolithotomy and flexible ureteroscopic lithotripsy was investigated in the treatment of partial staghorn calculi, and was observed to be more efficient compared with the conventional minimally invasive percutaneous nephrolithotomy monotherapy, without additional procedure-associated complications (30). In the current study, the results indicated that PMIS presented a superior performance to COS for the treatment of patients with fractures in the thoracic spine. It was also demonstrated that the anterolisthesis and neurological state were significantly improved in patients receiving PMIS as compared with those receiving COS. Notably, the study findings further suggested that there were significant differences between PMIS and COS as determined by the VAS score, JOA score and ASIA grade of the patient symptoms.

Although a previous review revealed that PMIS has a comparative effectiveness and safety with COS in the treatment of fractures of the thoracolumbar junction (12), further investigation on the effectiveness of PMIS in the treatment 
A

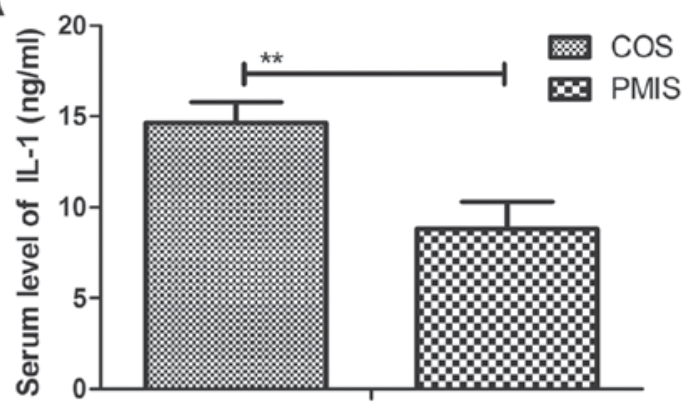

C

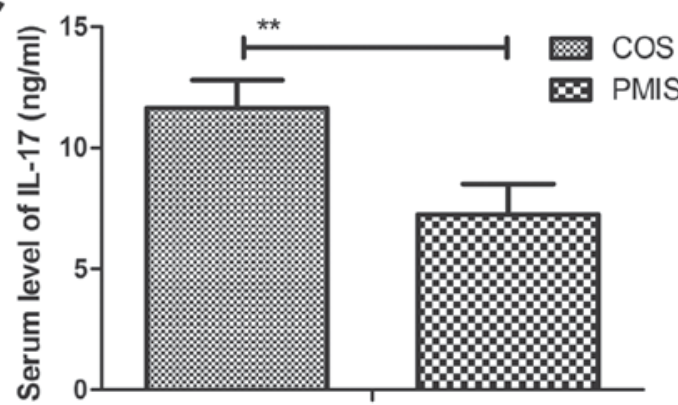

B

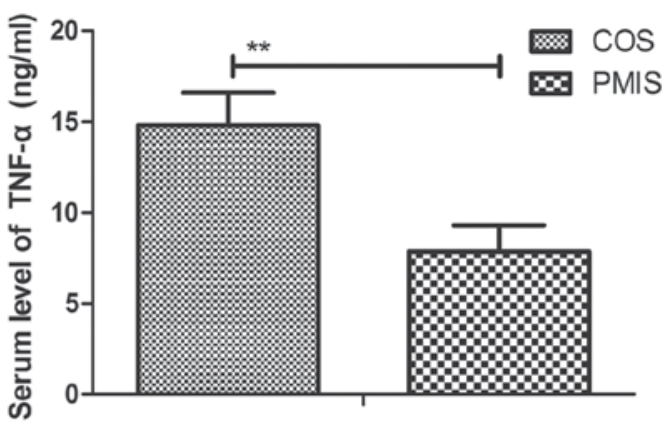

D

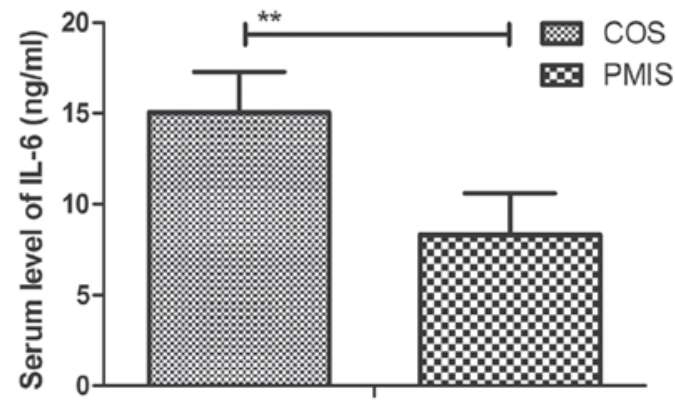

Figure 2. Comparison of inflammation between the thoracic spine fracture patients receiving PMIS or COS. The serum levels of (A) IL-1, (B) TNF- $\alpha$, (C) IL-17 and (D) IL-6 in patients with fractures in the thoracic spine were measured by ELISA subsequent to PMIS or COS. * P $<0.01$. PMIS, percutaneous minimally invasive surgery; COS, conventional open surgery; IL, interleukin; TNF- $\alpha$, tumor necrosis factor $\alpha$.
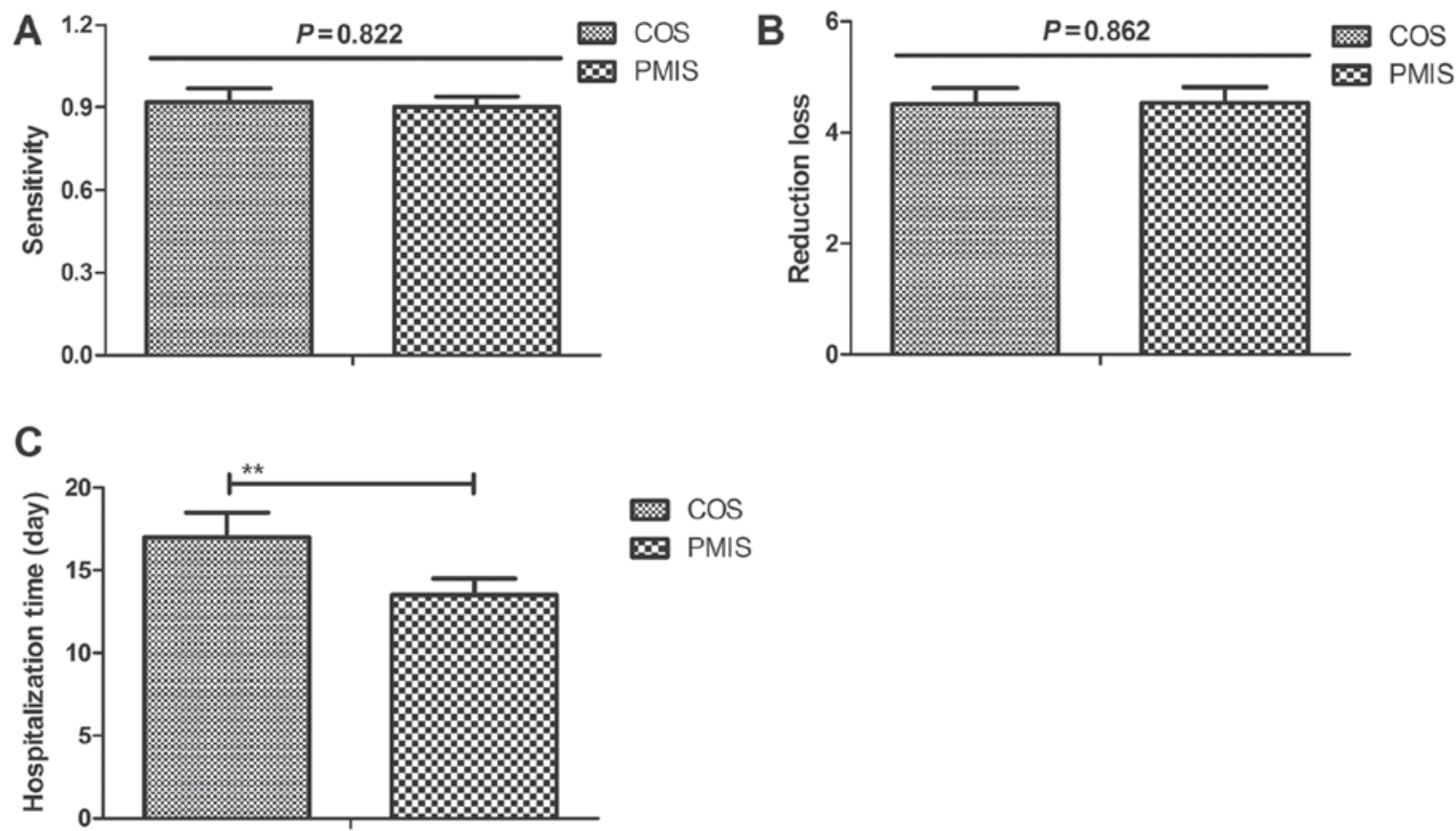

Figure 3. Comparison of (A) sensitivity, (B) mean values of reduction and correction loss, and (C) hospitalization time for fracture localization between the PMIS and COS patients with fractures in the thoracic spine. The sensitivity for fracture localization and the reduction loss were not significant different subsequent to PMIS or COS. Patients who underwent PMIS presented reduced hospitalization time compared with patients who underwent COS. ${ }^{* *} \mathrm{P}<0.01$. PMIS, percutaneous minimally invasive surgery; COS, conventional open surgery.

of thoracic spine fractures is required. In the current study, clinical analysis indicated that PMIS presents more benefits for patients with fractures in the thoracic spine, while the study also improved the understanding on the management of thoracic spine injuries. In addition, previous studies have demonstrated that posterior instruments of PMIS may improve the therapeutic efficacy for patients with fractures $(31,32)$. However, additional studies with larger samples and extended follow-ups are required to further assess the efficacy of this minimally invasive surgical technique for the treatment of thoracic spine 

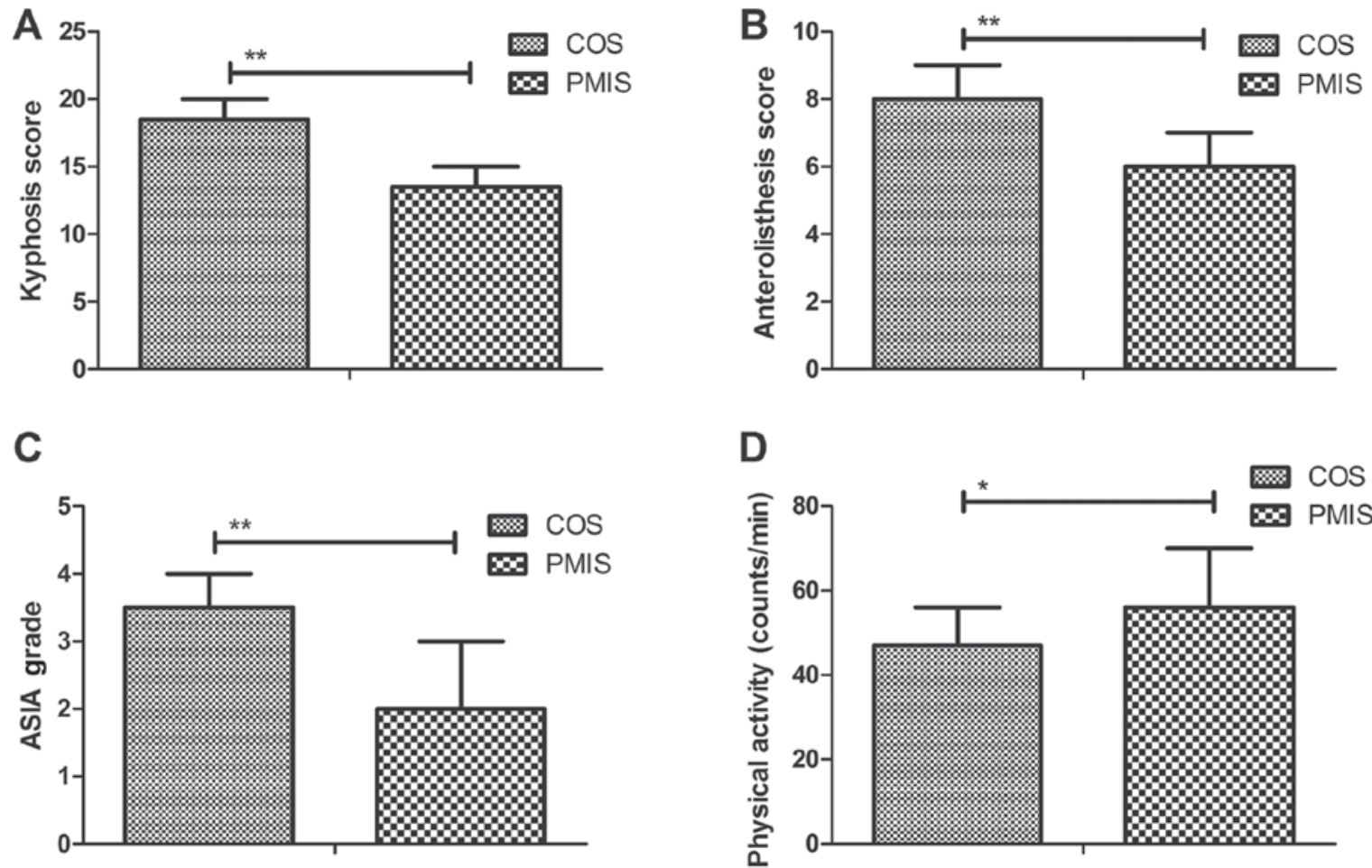

Figure 4. Comparison of physical activity between PMIS and COS patients with fractures in the thoracic spine. (A) The kyphosis rate, (B) anterolisthesis rate, and (C) neurological state (according to the ASIA grade) were markedly improved in patients who underwent PMIS as compared with those who underwent COS. (D) The physical activity results indicated that patients who underwent PMIS were more active compared with patients who underwent COS. "P<0.05 and ${ }^{* *} \mathrm{P}<0.01$. PMIS, percutaneous minimally invasive surgery; COS, conventional open surgery; ASIA, American Spinal Injury Association.
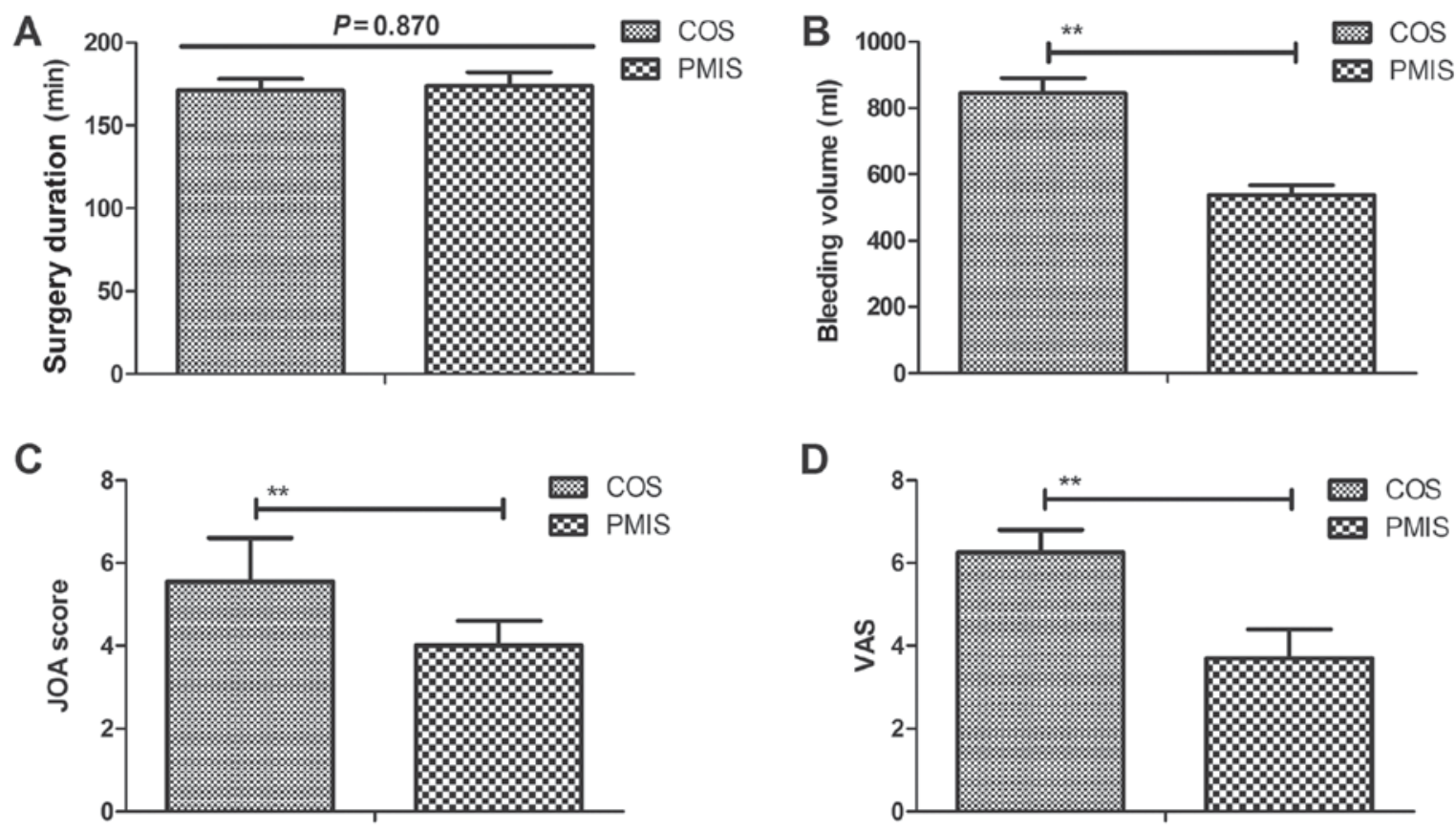

Figure 5. Comparison of physical condition between the PMIS and COS patients with fractures in the thoracic spine. (A) The surgery duration was similar for patients with fractures in the thoracic spine receiving PMIS and COS. (B) PMIS improved the bleeding volume during surgery for patients with fractures in the thoracic spine compared with COS. The mean (C) JAO and (D) VAS scores were decreased in patients with fractures in the thoracic spine who received PMIS compared with COS. ${ }^{* *} \mathrm{P}<0.01$. PMIS, percutaneous minimally invasive surgery; COS, conventional open surgery; VAS, visual analog scale; JOA, Japanese Orthopedic Association.

fracture $(33,34)$. In the present study, a large number of clinical patients were recruited to analyze the advantages of PMIS as compared with those of COS. However, long-term differences
( $>1$ year) between PMIS and COS in patients with fractures in the thoracic spine were not compared, which is a limitation of the current study. Additionally, the study was only able to 
provide short-term inflammation and neurologic data, and thus long-term results should be investigated in future studies.

In conclusion, the present study suggested that PMIS has a superior performance in patients with thoracic spine fractures as compared with COS. It is indicated that PMIS is more beneficial in decreasing inflammation and blood loss for the treatment of patients with fractures in the thoracic spine. However, further studies are required to verify these findings in clinical patients with fractures in the thoracic spine.

\section{References}

1. Beisse R and Verdú-López F: Current status of thoracoscopic surgery for thoracic and lumbar spine. Part 1: General aspects and treatment of fractures. Neurocirugia (Astur) 25: 8-19, 2014.

2. Božík M, Magala M, Heger T, Matejička D, Baka J and Šimko P: Pedicle screw fixation of thoracic spine fractures. Acta Chir Orthop Traumatol Cech 81: 140-151, 2014 (In Slovak).

3. van der Jagt-Willems HC, van Munster BC, Tulner LR and Lems WF: Geriatricians should screen for vertebral fractures in all individuals by performing X-rays of the thoracic spine. J Am Geriatr Soc 62: 2027-2029, 2014

4. Singh R, McD Taylor D, D'Souza D, Gorelik A, Page P and Phal P: Injuries significantly associated with thoracic spine fractures: A case-control study. Emerg Med Australas 21: 419-423, 2009.

5. Gross EA: Computed tomographic screening for thoracic and lumbar fractures: Is spine reformatting necessary? Am J Emerg Med 28: 73-75, 2010.

6. Ulmar B, Gühring M, Stuby F, Brunner A, Schmälzle T, Weise K and Badke A: Traumatic thoracic spine fractures: Inter- and intraobserver reliability of vertebral, local and segmental kyphosis in lateral X-rays. Z Orthop Unfall 147: 481-486, 2009 (In German)

7. Rosengart TK, Feldman T, Borger MA, Vassiliades TA Jr, Gillinov AM, Hoercher KJ, Vahanian A, Bonow RO, O'Neill W; American Heart Association Council on Cardiovascular Surgery and Anesthesia, et al: Percutaneous and minimally invasive valve procedures: A scientific statement from the American Heart Association Council on Cardiovascular Surgery and Anesthesia, council on clinical cardiology, functional genomics and translational biology interdisciplinary working group, and quality of care and outcomes research interdisciplinary working group. Circulation 117: 1750-1767, 2008.

8. Raffa GM, Pellegrini C, Lentini S, Perrotta S, Tancredi F, Gaeta R and Viganò M: Minimally invasive video-assisted surgery for iatrogenic aortic root-to-right atrium fistula after incomplete percutaneous occlusion of patent foramen ovale: Case report and review of the literature. J Card Surg 23: 75-78, 2008.

9. Ohuchi H, Kyo S, Asano H, Tanabe H, Yokote Y and Omoto R: Development and clinical application of minimally invasive cardiac surgery using percutaneous cardiopulmonary support Jpn J Thorac Cardiovasc Surg 48: 562-567, 2000.

10. Deo SV, Sharma V, Shah IK, Erwin PJ, Joyce LD and Park SJ: Minimally invasive direct coronary artery bypass graft surgery or percutaneous coronary intervention for proximal left anterior descending artery stenosis: A meta-analysis. Ann Thorac Surg 97: 2056-2065, 2014.

11. Kwan MK, Lee CK and Chan CY: Minimally invasive spinal stabilization using fluoroscopic-guided percutaneous screws as a form of palliative surgery in patients with spinal metastasis. Asian Spine J 10: 99-110, 2016.

12. Barbagallo GM, Yoder E, Dettori JR and Albanese V: Percutaneous minimally invasive versus open spine surgery in the treatment of fractures of the thoracolumbar junction: A comparative effectiveness review. Evid Based Spine Care J 3: 43-49, 2012.

13. Takami M, Yamada H, Nohda K and Yoshida M: A minimally invasive surgery combining temporary percutaneous pedicle screw fixation without fusion and vertebroplasty with transpedicular intracorporeal hydroxyapatite blocks grafting for fresh thoracolumbar burst fractures: prospective study. Eur J Orthop Surg Traumatol 24 (Suppl 1): S159-S165, 2014

14. Tu KK, Zhou XT, Tao ZS, Chen WK, Huang ZL, Sun T, Zhou Q and Yang L: Minimally invasive surgical technique: Percutaneous external fixation combined with titanium elastic nails for selective treatment of tibial fractures. Injury 46: 2428-2432, 2015.
15. Napier RJ and Nolan PC: Diagnosis of vertebral fractures in post-ictal patients. Emerg Med J 28: 169-170, 2011

16. Pavon JM, Sanders LL, Sloane R and Colón-Emeric C: Sensitivity of osteoporosis screening guidelines for eventual hip fracture in older male veterans. Bonekey Rep 3: 530, 2014

17. Kataoka H, Ikemoto T, Yoshimura A, Shibuya M, Goto K, Yamashita J, Morita K, Sakamoto J, Nakano J and Okita M: Association of early physical activity time with pain, activities of daily living, and progression of vertebral body collapse in patients with vertebral compression fractures. Eur J Phys Rehabil Med 53: 366-376, 2017.

18. Rahbek O, Jensen SL, Lind M, Penny JØ, Kallemose T, Jakobsen $\mathrm{T}$ and Troelsen A: Inferior reliability of VAS scoring compared with International Society of the Knee reporting system for abstract assessment. Dan Med J 64: pii: A5346, 2017.

19. Yonenobu K, Abumi K, Nagata K, Taketomi E and Ueyama K: Interobserver and intraobserver reliability of the japanese orthopaedic association scoring system for evaluation of cervical compression myelopathy. Spine (Phila Pa 1976) 26: 1890-1895, 2001.

20. Payer M, Smoll NR, Oezkan N and Tessitore E: Dynamic transpedicular stabilisation and decompression in single-level degenerative anterolisthesis and stenosis. Acta Neurochir (Wien) 156: 221-227, 2014.

21. van Middendorp JJ, Hosman AJ, Pouw MH; EM-SCI Study Group and Van de Meent H: ASIA impairment scale conversion in traumatic SCI: is it related with the ability to walk? A descriptive comparison with functional ambulation outcome measures in 273 patients. Spinal Cord 47: 555-560, 2009.

22. Ippolito E, Farsetti P, Boyce AM, Corsi A, De Maio F and Collins MT: Radiographic classification of coronal plane femoral deformities in polyostotic fibrous dysplasia. Clin Orthop Relat Res 472: 1558-1567, 2014

23. Bartley CE, Bastrom TP and Newton PO: Blood loss reduction during surgical correction of adolescent idiopathic scoliosis utilizing an ultrasonic bone scalpel. Spine Deform 2: 285-290, 2014.

24. Kreinest M, Schmahl D, Grützner PA and Matschke S: Trisegmental fusion by vertebral body replacement: Outcome following traumatic multisegmental fractures of the thoracic and lumbar spine. Unfallchirurg 121: 300-305, 2018 (In German).

25. Linhares D, Neves N, Ribeiro da Silva M and Almeida Fonseca J: Analysis of the cochrane review: Pedicle screw fixation for traumatic fractures of the thoracic and lumbar spine. Cochrane database syst rev. 2013;05:CD009073. Acta Med Port 29: 297-300, 2016 (In Portuguese).

26. Chiu KM, Lin TY, Chen JS and Chu SH: Percutaneous cardioplegia delivery using the miniport in minimally invasive mitral valve surgery. Interact Cardiovasc Thorac Surg 7: 342-343, 2008.

27. Zhang W, Li H, Zhou Y, Wang J, Chu T, Zheng W, Chen B and Li C: Minimally invasive posterior decompression combined with percutaneous pedicle screw fixation for the treatment of thoracolumbar fractures with neurological deficits: A prospective randomized study versus traditional open posterior surgery. Spine (Phila Pa 1976) 41 (Suppl 19): B23-B29, 2016.

28. Zeng BF: Minimally invasive surgery in fracture management. Chin Med J (Engl) 121: 1349-1351, 2008.

29. Pan YW, Wang XM and Pei XQ: Treatment of children supracondylar fracture of humerus with minimally invasive surgery. Zhongguo Gu Shang 22: 343, 2009 (In Chinese).

30. Wen J, Xu G, Du C and Wang B: Minimally invasive percutaneous nephrolithotomy versus endoscopic combined intrarenal surgery with flexible ureteroscope for partial staghorn calculi: A randomised controlled trial. Int J Surg 28: 22-27, 2016.

31. Wei FC: Minimally invasive surgery for vertebral fracture and spinal infection. Biomed J 36: 153, 2013.

32. Jing ZF, Zhao YY, Wang RG, Wang GZ and Teng LL: Minimally invasive surgery to treat severe acromioclavicular dislocation combined with coracoid process fracture. Zhongguo $\mathrm{Gu}$ Shang 23: 46-48, 2010.

33. Hu YM and Pang QJ: Effectiveness of manipulative reduction combined with minimally invasive surgery in the treatment of osteoporotic vertebral compression fracture: A meta-analysis. Zhongguo Gu Shang 28: 1042-1047, 2015.

34. Wang J, Zhou Y, Zhang ZF, Li CQ, Zheng WJ and Huang B: Minimally invasive transforaminal interbody fusion surgery for the old fracture of the thoracolumbar junction. J Spinal Disord Tech 27: E55-E60, 2014 